\title{
FE-simulation of the maxillary sinus floor augmentation
}

\author{
J. Lenz ${ }^{1}$, S. Rues ${ }^{1}$, H. P. Schierle ${ }^{1}$, K. Schweizerhof ${ }^{2}$ \\ \& H. J. Schindler ${ }^{1}$ \\ ${ }^{I}$ Research Group Biomechanics, Department of Mathematics, \\ University of Karlsruhe, Germany \\ ${ }^{2}$ Institute for Mechanics, University of Karlsruhe, Germany
}

\begin{abstract}
The sinus floor augmentation was put into practice about 20 years ago to provide the severely atrophic maxilla (alveolar ridge inappropriate for classical implantation) with dental implants. In the most frequently used surgical method a window-like lid is milled into the buccal bone shell adjacent to the paranasal sinus in the region of teeth $5-7$, and the bone lid is carefully flapped into the sinus without injuring the mucous membrane. Thus, a cavity is created into which either autologous bone material (e.g. from the chin or iliac crest) and/or bone substitute can be augmented to yield an adequately spacious support for (up to three) implants. After sufficient maturation of the graft (healing phase of several months) the implants can be connected to a superstructure. In this contribution the sinus floor is geometrically reproduced on the basis of a selfdeveloped three-dimensional model of the facial skull which contains all cortical and spongy structures. With the aid of the Finite Element Method (FEM) the distributions of stress, strain and displacement in the cortical and spongy bone as well as in the graft are analysed under different masticatory loads with special attention to the force deduction in the different structures. A variation of Young's modulus $\mathrm{E}_{\text {graft }}$ of the augmented material between 0.01 and $15 \mathrm{GPa}$ (cortical bone), thus simulating different degrees of maturation, proves that already about half of the external axial force is picked up by the graft when it reaches the maturation of spongy bone $(2 \mathrm{GPa})$. Furthermore, the analysis shows that for $\mathrm{E}_{\text {graft }}=2 \mathrm{GPa}$ the microstrains under average chewing forces fall into the physiological or mild overuse window according to Frost's Mechanostat Theory. Keywords: implantology, sinus lift, augmentation, bone remodeling, FEM.
\end{abstract}




\section{Introduction}

A successful incorporation of dental implants requires, among other preconditions (bone quality, distribution of implants, prosthesis design etc.), above all an adequate amount of bone in the vicinity of the site of implantation. Recently, in patients with atrophic jaws, augmentation of the alveolar ridge with autologous and/or heterogeneous graft material or distraction methods have been used in order to create an ample bony support for implants.
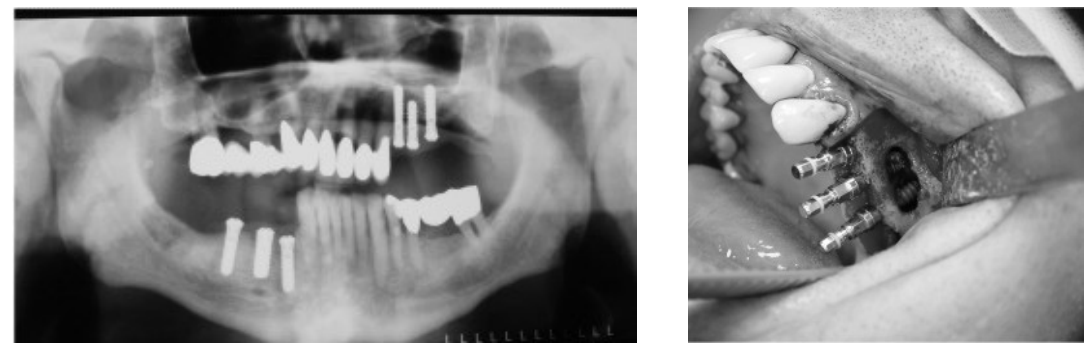

Figure 1: Radiograph of a severely atrophic maxilla with three implants in the patient's left sinus (left); bone window (right).

An alternative method for the severely atrophic maxilla (ridge height smaller than $\sim 5 \mathrm{~mm}$ ) is the so-called sinus floor augmentation (sinus lift method) [1-6]. In the most frequently performed surgical procedure a bone lid is milled into the buccal bone shell adjacent to the paranasal sinus approximately in the region of teeth $5-7$, and this bone lid is carefully flapped into the sinus without injuring the mucous membrane, as shown in fig. 1 and schematically in fig. 2 . The cavity thus generated can then be filled either with autologous graft material from the iliac crest or the chin and/or heterogeneous graft material. Such a structure can serve as an adequately extensive support for up to three implants.

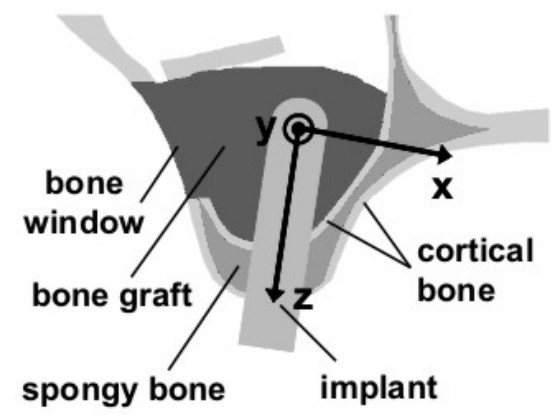

Figure 2: Scheme of the augmented sinus floor and used coordinate system.

For the maintenance of the bone/implant interface biomechanical factors (bone quality, distribution of the implants in the jaw, magnitude and direction of 
masticatory forces, prosthesis design etc.) play an important role [7]. According to Frost's Mechanostat Theory [8] mechanical forces may stimulate either bone apposition or bone resorption. Insufficient strains in the bony support around an implant cause disuse atrophy of the bone while excessive strains in the bone result in a necrosis (microfractures). Both phenomena may lead to implant failure. For that reason this investigation also addresses the distribution of (micro)strains in the bone graft as a function of its instantaneous stiffness.

\section{Materials and methods}

Fig. 3 shows the used FE-model of the sinus floor equipped with three splinted implants in region 5, 6 and 7 (omitting the augmented material). The cylindrical titanium implants have a diameter of $4 \mathrm{~mm}$, the insertion depth of the implants amounts to $\sim 12 \mathrm{~mm}$. The implants are tilted in palatal direction by $6^{\circ}$ to $8^{\circ}$ with respect to the saggital plane, and connected with a superstructure.

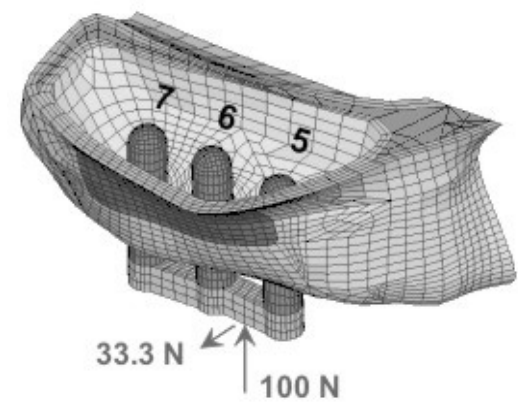

Figure 3: FE-model of the sinus floor (without bone graft) equipped with three implants, and loading of the superstructure.

Table 1 presents the average fraction of the contact (bonding) areas of the implants with the exterior cortical bone, spongy (trabecular, cancellous) bone, interior cortical bone (adjacent to the sinus floor), and the bone graft.

Table 1: Fraction of the surface of the implants in the different bone regions.

\begin{tabular}{|l|c|c|}
\hline region & surface $\left(\mathrm{mm}^{2}\right)$ & surface fraction (\%) \\
\hline exterior cortical bone & 6 & 4 \\
\hline spongy (trabecular) bone & 42 & 28 \\
\hline interior cortical bone & 7 & 5 \\
\hline bone graft & 95 & 63 \\
\hline
\end{tabular}

All materials involved were modelled as isotropic, homogeneous, linearelastic materials. The used Young's moduli and Poisson's ratios are given in Table 2. In order to study the effect of the degree of maturation of the bone graft on the stability of the implants, its Young's modulus $E_{\text {graft }}$ was varied between 
the limits $0.01 \mathrm{GPa}$ (simulating total resorption of the graft as well as a stiffness immediately after insertion) and $15 \mathrm{GPa}$ (total corticalization of the graft). The implant/bone interface was assumed to be perfect thus simulating full osseointegration. The ridge height of the alveolar bone in the FE model amounted to about $4 \mathrm{~mm}$.

Table 2: $\quad$ Material coefficients used in the simulations.

\begin{tabular}{|l|c|c|}
\hline material & Young's modulus $(\mathrm{GPa})$ & Poisson's ratio \\
\hline implant (titanium) & 110 & 0.3 \\
\hline splint (titanium) & 110 & 0.3 \\
\hline cortical bone & 15 & 0.3 \\
\hline spongy (trabecular) bone & 2 & 0.3 \\
\hline bone graft & $\begin{array}{c}\text { variation between } 0.01 \mathrm{GPa} \\
\text { and 15 GPa (cortical bone) }\end{array}$ & 0.3 \\
\hline
\end{tabular}

Fig. 4 shows (left) the radiograph of a frontal intersection through a skull (one side endowed with a tooth) and an intersection through the complete model of the facial skull used in the simulations (right). For the support of the model all nodes of the plane upper intersections were fixed. The FE-mesh comprised $\sim 124,400$ (mostly hexahedral) elements corresponding to $\sim 82,000$ nodes. The computations were carried out with the commercial program ANSYS 7.1.
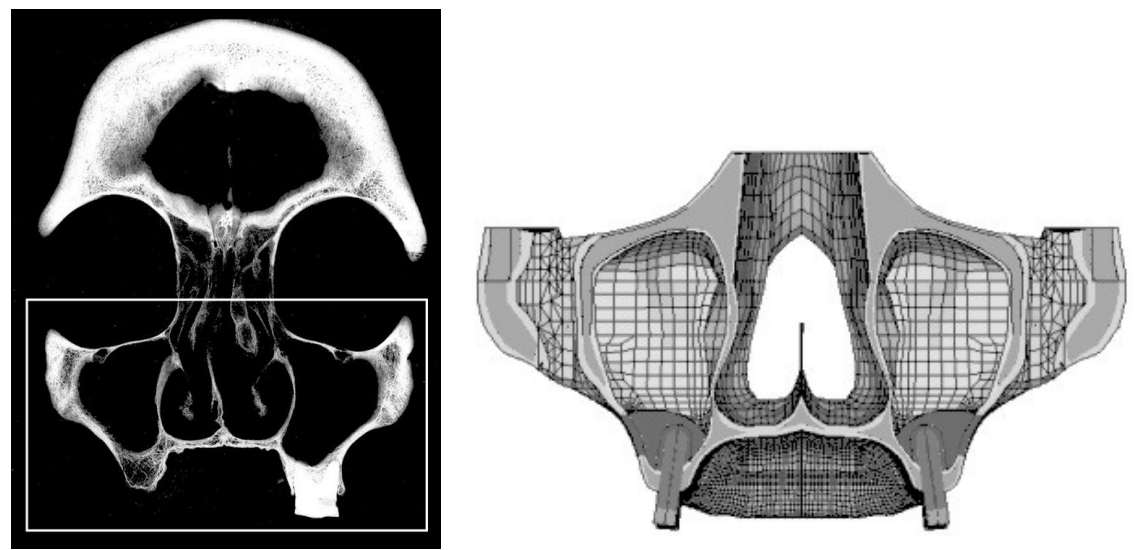

Figure 4: Frontal intersection through a skull showing the nasal cavities (left), and the complete FE-model displaying the augmented sinus (right).

Two load cases were considered: (1) a force of $100 \mathrm{~N}$ in axial direction of the implant in region 6 (load case LC1), and (2) a combination of an axial force of $100 \mathrm{~N}$ and a horizontal force of $33.3 \mathrm{~N}$ in buccal direction (load case LC2), both 
loads being transferred (cf. fig. 3) to the connector in the centre between implants 5 and 6 (in the simulations of horizontal forces the buccal direction delivered the highest stresses). Since masticatory forces lie in the range of $\sim 50$ $300 \mathrm{~N}$, depending on the kind and texture of the bole, both loads can be considered as examples of moderate to average chewing forces [9].

\section{Results}

For the two considered load conditions, table 3 summarizes the maximum tensile stresses found in the four bony structures for the values $\mathrm{E}_{\text {graft }}=0.01 \mathrm{GPa}, 2 \mathrm{GPa}$ (spongy bone), and $15 \mathrm{GPa}$ (cortical bone). It becomes evident that with growing $\mathrm{E}_{\text {graft }}$ the stresses in the cortical and spongy sections essentially decrease whereas those in the graft increase moderately. In view of stress values in the bone around single implants presented in the literature [10], all stresses can be considered as atraumatically tolerable for the maxilla. It becomes also clear that load case LC2 (superimposed horizontal force on the splint) leads to considerably higher stresses in the cortical and spongy bone than LC1 (purely axial force).

Table 3: $\quad$ Maximum tensile stresses in the bony structures.

\begin{tabular}{|l|ccc|ccc|}
\hline & \multicolumn{3}{|c|}{$\sigma_{I, \max }(\mathrm{MPa}) ; \mathrm{LC} 1$} & \multicolumn{3}{c|}{$\sigma_{\mathrm{I}, \max }(\mathrm{MPa}) ; \mathrm{LC} 2$} \\
\hline $\mathrm{E}_{\text {graft }}(\mathrm{GPa})$ & 0.01 & 2 & 15 & 0.01 & 2 & 15 \\
\hline exterior cortical bone & 5.4 & 2.5 & 1.5 & 11.5 & 8.2 & 9.5 \\
\hline spongy (trab.) bone & 3.5 & 0.5 & 0.4 & 5.9 & 1.1 & 0.8 \\
\hline interior cortical bone & 9.5 & 2.0 & 0.6 & 16.0 & 2.8 & 1.4 \\
\hline bone graft & 0.0 & 0.5 & 0.7 & 0.0 & 0.8 & 0.7 \\
\hline
\end{tabular}

As shown in table 4, the maximum compressive stresses in the bony structures tend to result in higher values which holds especially for the exterior cortical shell. Again, stresses decrease with increasing $\mathrm{E}_{\text {graft }}$, and LC2 delivers considerably higher stress values than LC1.

Table 4: $\quad$ Maximum compressive stresses in the bony structures.

\begin{tabular}{|l|ccc|ccc|}
\hline & \multicolumn{3}{|c|}{$\sigma_{\text {III,max }}(\mathrm{MPa}) ; \mathrm{LC} 1$} & \multicolumn{3}{c|}{$\sigma_{\text {III,max }}(\mathrm{MPa}) ; \mathrm{LC} 2$} \\
\hline $\mathrm{E}_{\text {graft }}(\mathrm{GPa})$ & 0.01 & 2 & 15 & 0.01 & 2 & 15 \\
\hline exterior cortical bone & -23.4 & -13.8 & -9.2 & -32.4 & -15.8 & -13.9 \\
\hline spongy (trab.) bone & -2.5 & -0.9 & -0.6 & -4.6 & -1.1 & -1.0 \\
\hline interior cortical bone & -13.3 & -4.8 & -2.9 & -22.1 & -6.8 & -5.0 \\
\hline bone graft & 0.0 & -0.8 & -1.8 & 0.0 & -1.2 & -2.6 \\
\hline
\end{tabular}

Supplementary, fig. 5 shows for LC1 the distribution of maximum tensile stresses in the bony structures around the implants for $\mathrm{E}_{\text {graft }}=2 \mathrm{GPa}$ (view to the buccal side). 


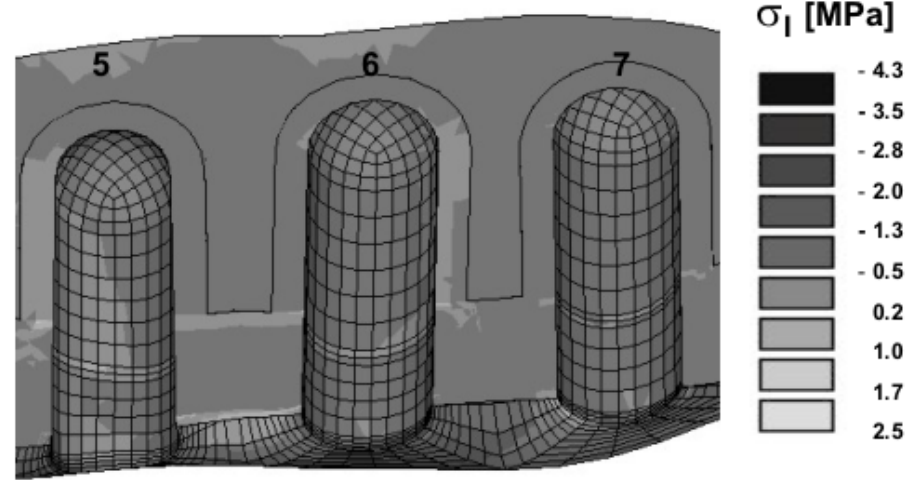

Figure 5: $\quad$ Distribution of maximum tensile stresses in load case LC1.

The distribution of maximum compressive stresses in LC2 for $\mathrm{E}_{\text {graft }}=2 \mathrm{GPa}$ is given in fig. 6 (view to the buccal side).
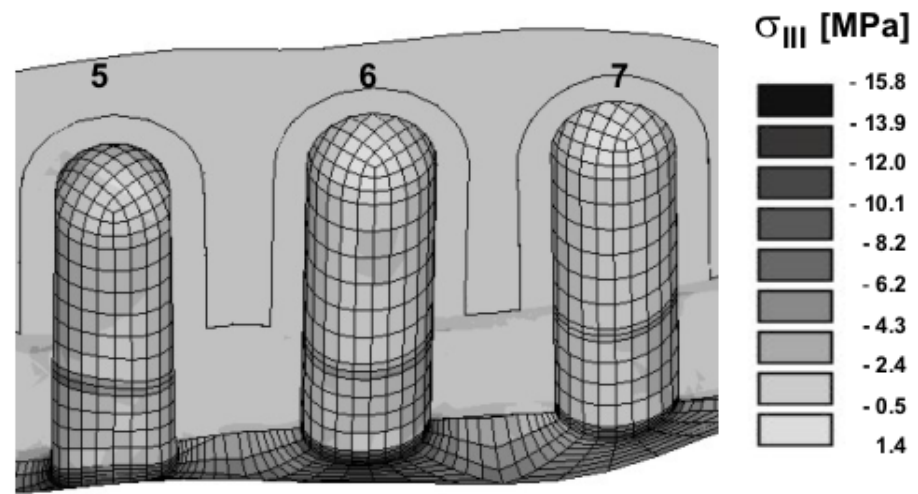

Figure 6: Distribution of maximum compressive stresses in load case LC2.

Presumably more interesting is the question, how much of the force applied to each implant is taken up by the different bone segments. Fig. 7 presents, for the load case $\mathrm{LC} 1$, the quotient of axial reaction forces $\mathrm{F}_{\mathrm{z} \text {,bone }} / \mathrm{F}_{\mathrm{z}}\left(\mathrm{F}_{\mathrm{z} \text {,bone }}\right.$ : axial force adopted by the correspondent bone segment, $F_{z}$ : total axial force on the considered implant) as a function of Young's modulus $E_{\text {graft }}$ of the augmented material.

With increasing stiffness $E_{\text {graft }}$ the bone graft takes up most of the axial reaction force component acting on each implant. As seen from fig. 7, the graft when homogeneously reaching the maturation of spongy bone $\left(\mathrm{E}_{\text {graft }}=2 \mathrm{GPa}\right)$, already picks up approximately half of the axial reaction force in all implants. This result supports the assumption that a mechanically firm incorporation of an implant in the augmented sinus might already be achieved if the graft reaches the stiffness of spongy bone. 


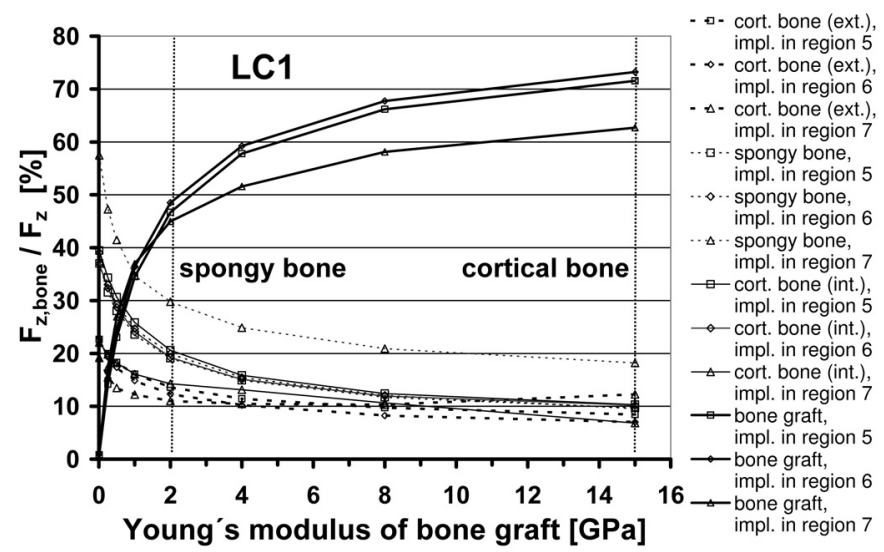

Figure 7: Influence of Young's modulus of the bone graft on the axial reaction forces in the different bone segments.

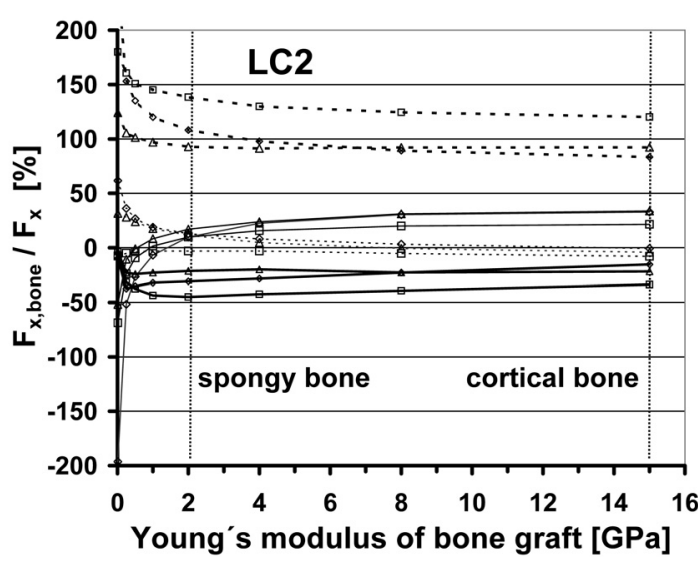

$$
\begin{aligned}
& \text { - घ- cort. bone (ext.), } \\
& \text { impl. in region } 5 \\
& \text { cort. bone (ext.) } \\
& \text { impl. in region } 6 \\
& \Delta \text { - cort. bone (ext.) } \\
& \text { impl. in region } 7 \\
& \text {... spongy bone, } \\
& \text { impl. in region } 5 \\
& \text {-spongy bone, } \\
& \text { impl. in region } 6 \\
& \text {-spongy bone, } \\
& \text { impl. in region } 7 \\
& \text { ๑-cort. bone (int.) } \\
& \text { impl. in region } 5 \\
& \checkmark \text { cort. bone (int.), } \\
& \text { impl. in region } 6 \\
& \triangle \text { cort. bone (int.), } \\
& \text { impl. in region } 7 \\
& \text { —bone graft, } \\
& \text { impl. in region } 5 \\
& \longrightarrow \text { bone graft, } \\
& \text { impl. in region } 6 \\
& \text { impl. in region } 7
\end{aligned}
$$

Figure 8: Influence of Young's modulus of the bone graft on the horizontal reaction forces in the different bone segments.

Fig. 8 shows the corresponding results for the horizontal (buccal) reaction

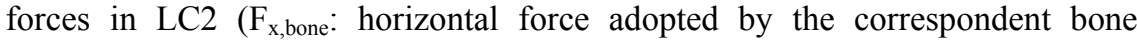
segment, $\mathrm{F}_{\mathrm{x}}$ : horizontal total force on the considered implant). It becomes evident that horizontal forces, on the contrary, are predominantly taken up by the exterior cortical shell. In this load case the horizontal component also produces a high torque which must be balanced by a couple of forces. Therefore, in addition to the high horizontal force applied to the exterior cortical shell, the other bone structures experience forces compensating for this effect. For small values of 
$E_{\text {graft }}$ very high forces are applied to the exterior and interior cortical shell (cf. fig. 8) because of the small moment arm. These forces decrease rapidly with increasing Young's modulus of the graft (and thereby also growing moment arm). Once Young's modulus of the graft reaches the value of spongy bone, further maturation does not lead to an increase in its adoption of horizontal reaction forces.

According to Frost's Mechanostat Theory [8] the effect of local microstrain $\left(1 \mu \varepsilon=10^{-6}\right)$ on bone can be divided into four intervals:

(1) disuse window for $0<\mu \varepsilon<\sim(50$ - 200) in which bone resorption $>$ bone formation,

(2) physiological window for $\sim(50-200)<\mu \varepsilon<\sim(1500-2500)$ in which resorption $=$ formation (homeostasis),

(3) mild overuse window for $\sim(1500-2500)<\mu \varepsilon<\sim 4000$ in which bone formation $>$ resorption, and

(4) damage window for $\mu \varepsilon>\sim 4000$ in which microdamage and increased bone resorption occur.

Analysis of the distribution of microstrain in the bone graft under the given loads as a function of $\mathrm{E}_{\text {graft }}$ delivers the following results:

(1) For $\mathrm{E}_{\text {graft }}=0.01 \mathrm{GPa}$ (very soft graft immediately after augmentation) average microstrain values lie in the range of $\mu \varepsilon \approx 200$ - 500 with maximum microstrains of $\mu \varepsilon_{\mathrm{I}} \approx 2800$ and $\mu \varepsilon_{\mathrm{III}} \approx-2500$ in the immediate vicinity of the implants.

(2) For $\mathrm{E}_{\text {graft }}=2 \mathrm{GPa}$ (Young's modulus of spongy bone) average microstrain values amount to $\mu \varepsilon \approx 100-200$ with maximum values of $\mu \varepsilon_{\mathrm{I}} \approx$ 350 and $\mu \varepsilon_{\text {III }} \approx-650$ which again occur at the implant/graft interface.

(3) For $\mathrm{E}_{\text {graft }}=15 \mathrm{GPa}$ (Young's modulus of cortical bone) average microstrain values amount to $\mu \varepsilon<100$ with maximum values of $\mu \varepsilon_{\text {I }} \approx 110$ and $\mu \varepsilon_{\text {III }} \approx-100$ at the implant/graft interface.

Taking into account that realistic masticatory loads are about twice as high as those used in this investigation which leads to about doubled microstrains, the following conclusions may be drawn:

(1) For $\mathrm{E}_{\text {graft }}=0.01 \mathrm{GPa}$ the maximum microstrains fall into the damage window. Since the presented values were computed under the assumption of direct loading of the splint, this might support the hypothesis that implants in the sinus should not be directly loaded immediately after insertion. Rather a healing phase (with implants hidden underneath the mucosa) seems to be advisable during which the bone graft experiences solely strains and stresses due to functional deformations of the maxilla. 
(2) For $\mathrm{E}_{\text {graft }}=2 \mathrm{GPa}$ the maximum microstrains fall into the physiological window (bone formation and bone apposition are in equilibrium; homeostasis) or at most into the mild overuse window.

(3) For $\mathrm{E}_{\text {graft }}=15 \mathrm{GPa}$ the maximum microstrains fall into the disuse window. This might indicate that the bone graft possibly never reaches the degree of maturation corresponding to cortical bone.
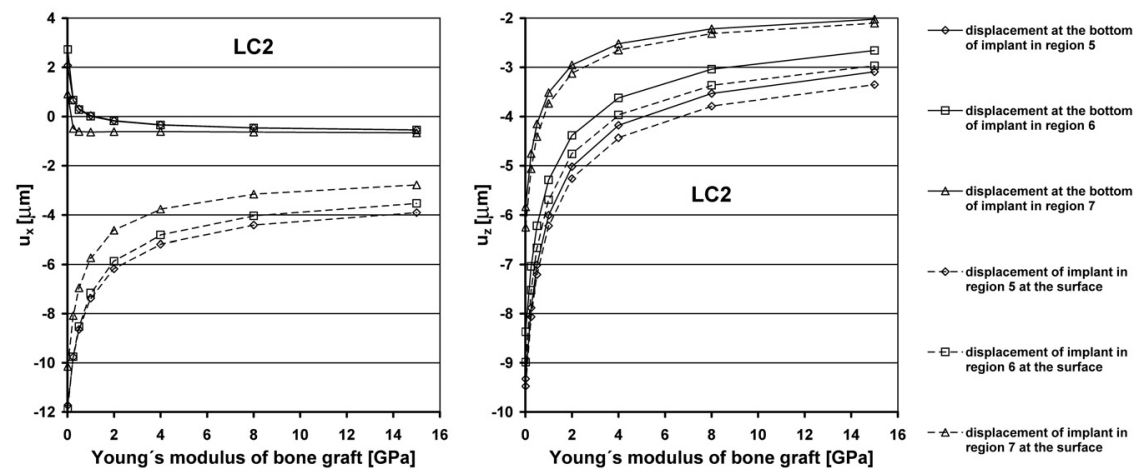

Figure 9: Axial (left) and lingual (right) displacements, $\mathrm{u}_{\mathrm{x}}$ and $\mathrm{u}_{\mathrm{z}}$, of the implant as a function of Egraft in load case LC2.

Fig. 9 shows the axial and lingual displacement components, $u_{x}$ and $u_{z}$, of the implant as a function of Young's modulus $\mathrm{E}_{\text {graft }}$ for the load case LC2 (coordinate system given in fig. 2). The displacement components $\mathrm{u}_{\mathrm{y}}$ are comparatively small.

Only one preceding publication [11] is known to the authors which deals with the development of a FE-model in connection with the sinus floor augmentation. In that paper only the sinus floor, and not the complete sinus including the surrounding bony structures, was modelled. Moreover, a quasi-two-dimensional model was employed, and only a single implant in the sinus floor considered. When trying to simulate this situation under identical loads as closely as possible with our three-dimensional model we are led to stresses and displacements which differ from those in [11] by $100 \%$ and more.

\section{Conclusions}

The results of the analysis let us hypothesize that a sufficiently firm incorporation of implants in the augmented sinus floor is secured if the graft reaches a degree of maturation corresponding to that of spongy (cancellous, trabecular) bone. Additionally, applying Frost's Mechanostat Theory, the microstrain values generated in the graft under realistic masticatory loads, fall into the physiological window or at most into the mild overuse window if Young's modulus of the graft complies approximately with that of spongy bone. Furthermore, the results advise that a healing phase should be allotted, because immediate loading of the 
implants could lead to damage in the graft due to overloading. Besides, as seen from table 4, especially the load case LC2 would lead to very high stresses in the cortical bone sections for small values $\mathrm{E}_{\text {graft }}$ (early loading).

\section{References}

[1] Misch, C.E., Maxillary sinus augmentation for endostial implants: Organized alternative treatment plans. International Journal of Oral Implantology, 4, pp. 49-58, 1987.

[2] Summers, R.B., Sinus floor elevation with osteotomes. Journal of Esthetic Dentistry, 10, pp. 164-171, 1998.

[3] Tatum, H., Maxillary and sinus implant reconstruction. Dental Clinics of North America, 30, pp. 207-229, 1986.

[4] Zitzmann, N.U. \& Schärer, P., Sinus elevation procedures in the resorbed posterior maxilla. Comparison of crestal and lateral approaches. Oral Surgery, Oral Medicine, Oral Pathology and Endodontics, 85, pp. 8-17, 1998.

[5] Jenssen, J., Krantz-Simonsen, E. \& Sindet-Pederssen, S., Reconstruction of the severely resorbed maxilla with bone grafting and osseointegrated implants. Journal of Oral Maxillofacial Surgery, 48, pp. 27-32, 1990.

[6] Ellegaard, B., Petersen, J.K. \& Stoltze, K., Implant therapy involving maxillary sinus lift in periodontally compromised patients. Clinical Oral Implants Research, 8, pp. 303-315, 1997.

[7] Skalak, R., Biomechanical considerations in osseointegrated prostheses. Journal of Prosthetic Dentistry, 49, pp. 843-848, 1983.

[8] Frost, H.M., The mechanostat: a proposed pathogenic mechanism of osteo-poroses and the bone mass effects of mechanical and nonmechanical agents. Bone and Mineral, 12, pp. 73-85, 1987.

[9] Schindler, H.J., Stengel, E. \& Spiess, W.E.L., Elektromyographische Aktivität, Kraftentwicklung und Kinematik beim Kauen fester Nahrungsstrukturen. Deutsche Zahnärztliche Zeitschrift, 53, pp. 551-556, 1998.

[10] Lenz, J., Freischläger, C., Rong, Q., Schweizerhof, K., Schindler, H.J. \& Riediger, D., Zum Risiko der Implantatversorgung bei Bruxismus. Eine Beanspruchungsanalyse mittels Finiter Elemente. Zeitschrift für Zahnärztliche Implantologie, 17, pp. 64-70, 2001.

[11] Tepper, G., Haas, R., Zechner, W., Krach, W. \& Watzek, G., Threedimensional finite element analysis of implant stability in the atrophic posterior maxilla. Clinical Oral Implants Research, 13, pp. 657-665, 2001. 